\title{
Helicoidal Surfaces in Galilean Space With Density
}

\author{
Safaa Mosa ${ }^{1,2}$ and Mervat Elzawy ${ }^{3,4 *}$ \\ ${ }^{1}$ Department of Mathematics, College of Science, University of Bisha, Bisha, Saudi Arabia, ${ }^{2}$ Mathematics Department, \\ Faculty of Science, Damanhour University, Damanhour, Egypt, ${ }^{3}$ Mathematics Department, Faculty of Science, Tanta \\ University, Tanta, Egypt, ${ }^{4}$ Mathematics Department, College of Science, Taibah University, Medina, Saudi Arabia
}

In this paper, we construct helicoidal surfaces in the three dimensional Galilean space $G^{3}$. The First and the Second Fundamental Forms for such surfaces will be obtained. Also, mean and Gaussian curvature given by smooth functions will be derived. We consider the Galilean 3-space with a linear density $e^{\phi}$ and construct a weighted helicoidal surfaces in $G^{3}$ by solving a second order non-linear differential equation. Moreover, we discuss the problem of finding explicit parameterization for the helicoidal surfaces in $G^{3}$.

M.S.C.2010: 53A35, 51A05

Keywords: Galilean space, helicoidal surfaces, density, mean curvature, Gauss curvature, weight helicoidal surface

OPEN ACCESS

Edited by:

Yang-Hui He,

City University of London,

United Kingdom

Reviewed by:

Yilun Shang,

Northumbria University,

United Kingdom

Praveen Agarwal,

Anand International College of

Engineering, India

*Correspondence:

Mervat Elzawy

mervatelzawy@science.tanta.edu.eg;

mrzawy@taibahu.edu.sa

Specialty section:

This article was submitted to

Mathematical Physics,

a section of the journal

Frontiers in Physics

Received: 27 November 2019

Accepted: 09 March 2020

Published: 31 March 2020

Citation:

Mosa S and Elzawy M (2020)

Helicoidal Surfaces in Galilean Space

With Density. Front. Phys. 8:81.

doi: 10.3389/fphy.2020.00081

\section{INTRODUCTION}

Due to its applications in probability and statistics, the study of manifolds with density has increased in the last years after Morgan's published his paper "Manifolds with density" [1]. As a new field in geometry, manifolds with density appear in different ways in mathematics, for example as quotients of Riemannian manifolds or as Gauss space [2].

Helicoidal surface is a natural generalization of rotation surface, of which many excellent works have been done, such as Kenmotsu [3].

For helicoidal surface in $R^{3}$, the cases with prescribed mean curvature or Gauss curvature have been studied by Baikoussis and Koufogiorgos [4]. Also, helicoidal surfaces in three dimensional Minkowski space has been considered by Beneki et al. [5]. A kind of helicoidal surface in 3-dimensional Minkowski space was constructed by Ji and Hou [6].

Construction of helicoidal surfaces in Euclidean space with density by solving second-order non-linear ordinary differential equation with weighted minimal helicoidal surface was introduced in Kim et al. [7]. For weighted type integral inequalities, one can see Agarwal et al. [8].

Mean and Gaussian curvature for surfaces are one of the main objects, which have geometers interest for along time. A manifold with density is a Riemannian manifold $M^{n}$ with a positive function $e^{\phi}$, known as density, used to weight volume and hypersurface area [2, 9]. A nice example of manifolds with density is Gauss space, the Euclidean space with Gaussian probability density $(2 \pi)^{\frac{-n}{2}} e^{\frac{-r^{2}}{2}}$, which is very useful to probabilists [2].

On a manifold with density $e^{\phi}$, the weighted mean curvature of a hypersurface with unit normal $N$ is defined by

$$
H_{\phi}=H-\frac{1}{n} \frac{d \phi}{d N}
$$

where $H$ is the Riemannian mean curvature of the hypersurface [9]. The weighted mean curvature $H_{\phi}$ of a surface in $E^{3}$ with density $e^{\phi}$ was introduced by Gromov [10], and it is a natural generalization of the mean curvature $H$ of a surface. The curvature concept is not confined to 
continuous space, it has been intensively studied in discrete mathematics including networks, for more details one can see Shang [11].

A surface with $H_{\phi}=0$ is known as a weighted minimal surface or a $\phi$-minimal surface in $E^{3}$ [12]. For more details about manifolds with density and other relative topics, we refer the reader to $[1-3,5-7,9,10,13-16]$. In particular, Yoon et al. [17] studied rational surfaces in Pseudo-Galilean space with a loglinear density and investigated $\phi-$ minimal rotational surfaces. Also, they classified the weighted minimal helicoidal surfaces in the Euclidean space $E^{3}$ [7].

The purpose of this paper is to construct helicoidal surface in Galilean space $G^{3}$. Firstly, we choose orthonormal basis as the coordinate frame and define helicoidal surface with density. The first fundamental form $d s^{2}$, the second fundamental form $I I$, the Gaussian and Mean curvature of helicoidal surface will be obtained in section 3. Secondly in section 4, we prescribed the parametrization of a weighted mean curvature $H_{\phi}=H-\frac{1}{2}<$ $N, \nabla \phi>$ and solving the non-linear differential equation.

\section{PRELIMINARIES}

In this part, we give a brief review of curves and surfaces in the Galilean space $G^{3}$. For more details, one can see $[12,14-16,18]$.

The Galilean 3 -space $G^{3}$ can be defined in the threedimensional real projective space $P_{3}(R)$ and its absolute figure is an ordered triple $\{\rho, f, I\}$, where $\rho$ is the ideal (absolute) plane, $f$ a line in $\rho$ and $I$ is the fixed elliptic involution of the points of $f$. We introduce homogeneous coordinates in $G^{3}$ in such a way that the absolute plane $\rho$ is given by $x_{o}=0$, the absolute line $f$ by $x_{o}=x_{1}=0$ and the elliptic involution by

$$
\left(0: 0: x_{2}: x_{3}\right) \rightarrow\left(0: 0: x_{3}:-x_{2}\right)
$$

A plane is said to be Euclidean if it contains $f$, otherwise it is called isotropic, i.e., the planes $x=$ const. are Euclidean, in particular the plane $\rho$. Other planes are isotropic.

The Galilean distance between the points $Q_{i}=\left(r_{i}, s_{i}, t_{i}\right)$, $(i=1,2)$ is given by

$$
d\left(Q_{1}, Q_{2}\right)= \begin{cases}\left|r_{2}-r_{1}\right|, & \text { if } r_{1} \neq 0 \text { or } r_{2} \neq 0 \\ \sqrt{\left(s_{2}-s_{1}\right)^{2}+\left(t_{2}-t_{1}\right)^{2}}, & \text { if } r_{1}=0 \text { and } r_{2}=0 .\end{cases}
$$

Moreover, the distance in the Euclidean space between $Q_{1}$ and $Q_{2}$ is given by

$$
d\left(Q_{1}, Q_{2}\right)=\sqrt{\left(r_{2}-r_{1}\right)^{2}+\left(s_{2}-s_{1}\right)^{2}+\left(t_{2}-t_{1}\right)^{2}}
$$

The Galilean scalar product between two vectors $P=\left(p_{1}, p_{2}, p_{3}\right)$ and $Q=\left(q_{1}, q_{2}, q_{3}\right)$ is defined by

$$
<P, Q>_{G}= \begin{cases}p_{1} q_{1}, & \text { if } p_{1} \neq 0 \text { or } q_{1} \neq 0 \\ p_{2} q_{2}+p_{3} q_{3}, & \text { if } p_{1}=0 \text { and } q_{1}=0 .\end{cases}
$$

For this, the Galilean norm of a vector $P$ is $\|P\|=\sqrt{\langle P, P\rangle_{G}}$. Moreover, the cross product in the Galilean space is defined by

$$
<P \times_{G} Q>=\left(0,\left|\begin{array}{ll}
p_{1} & p_{3} \\
q_{1} & q_{3}
\end{array}\right|,\left|\begin{array}{ll}
p_{1} & p_{2} \\
q_{1} & q_{2}
\end{array}\right|\right)
$$

A vector $P=\left(p_{1}, p_{2}, p_{3}\right)$ is said to be isotropic if $p_{1}=0$, otherwise it is known as non-isotropic. The following definitions will be helpful [19].

Definition 1. Let $a=\left(1, y_{2}, y_{3}\right)$ and $b=\left(1, z_{2}, z_{3}\right)$ be two unit non-isotropic vectors in general position in $G^{3}$. Then an angle $\theta$ between $a$ and $b$ is given by

$$
\theta=\sqrt{\left(z_{2}-y_{2}\right)^{2}+\left(z_{3}-y_{3}\right)^{2}}
$$

Definition 2. An angle $\psi$ between a unit non-isotropic vector $a=\left(1, y_{2}, y_{3}\right)$ and an isotropic vector $c=\left(0, z_{2}, z_{3}\right)$ in $G^{3}$ is given by

$$
\psi=\frac{y_{2} z_{2}+y_{3} z_{3}}{\sqrt{z_{2}^{2}+z_{3}^{2}}}
$$

Definition 3. An angle $\eta$ between two isotropic vectors $c=$ $\left(0, y_{2}, y_{3}\right)$ and $d=\left(0, z_{2}, z_{3}\right)$ parallel to the Euclidean plane in $G^{3}$ is equal to the Euclidean angle between them. Namely,

$$
\cos \eta=\frac{y_{2} z_{2}+y_{3} z_{3}}{\sqrt{y_{2}^{2}+y_{3}^{2}} \sqrt{z_{2}^{2}+z_{3}^{2}}}
$$

Definition 4. The curve $\alpha(t)=(x(t), y(t), z(t))$ in the Galilean space $G^{3}$ is said to be admissible if it has no inflection points

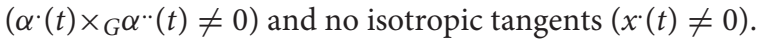

Let $C$ be an open subset of $R^{2}$ and $M$ a surface in $G^{3}$ parameterized by

$$
r: C \subseteq R^{2} \rightarrow G^{3}, r(u, v)=(x(u, v), y(u, v), z(u, v))
$$

In order to specify the partial derivatives we will denote:

$$
x_{u}=\frac{\partial x}{\partial u}, x_{v}=\frac{\partial x}{\partial v} \text { and } x_{u v}=\frac{\partial^{2} x}{\partial u \partial v}
$$

Then $r$ is satisfied admissibility criteria if no where it has Euclidean tangent planes. The first fundamental form is given by

$$
d s^{2}=\left(g_{1} d u+g_{2} d v\right)^{2}+\varepsilon\left(h_{11} d u^{2}+2 h_{12} d u d v+h_{22} d v^{2}\right)
$$

where $g_{1}=x_{u}=\frac{\partial x}{\partial u}, g_{2}=x_{v}=\frac{\partial x}{\partial v}$, and $h_{11}=y_{u}^{2}+z_{u}^{2}$, $h_{22}=y_{v}^{2}+z_{v}^{2}, h_{12}=y_{u} y_{v}+z_{u} z_{v}$, also

$$
\varepsilon=\left\{\begin{array}{l}
0, \text { if the direction } d u: d v \text { is non-isotropic } \\
1, \text { if the direction } d u: d v \text { is isotropic. }
\end{array}\right.
$$

Now, consider the function

$$
\omega=\left\|r_{u} \times r_{v}\right\|=\sqrt{\left(x_{u} z_{v}-x_{v} z_{u}\right)^{2}+\left(x_{v} y_{u}-x_{u} y_{v}\right)^{2}}
$$

hence the isotropic unit normal vector field $N$ of the surface $r=r(u, v)$ is given by

$$
N=\frac{r_{u} \times r_{v}}{\left\|r_{u} \times r_{v}\right\|}=\frac{1}{\omega}\left(0, x_{u} z_{v}-x_{v} z_{u}, x_{v} y_{u}-x_{u} y_{v}\right)
$$


The second fundamental form is obtained by

$$
I I=L_{11} d u^{2}+2 L_{12} d u d v+L_{22} d v^{2}
$$

such that

$$
\begin{aligned}
L_{i j} & =\frac{1}{g_{1}}\left(g_{1}\left(0, y_{i j}, z_{i j}\right)-\left(g_{i}\right)_{j}\left(0, y_{u}, z_{u}\right)\right) \cdot N \\
& =\frac{1}{g_{2}}\left(g_{2}\left(0, y_{i j}, z_{i j}\right)-\left(g_{i}\right)_{j}\left(0, y_{v}, z_{v}\right)\right) \cdot N
\end{aligned}
$$

where $i, j=u, v$.

Note that the dot “." denotes the Euclidean scalar product. Therefore, the Gaussian and mean curvature are given by.

$$
K=\frac{L_{11} L_{22}-L_{12}^{2}}{\omega^{2}} \text { and } H=\frac{g_{2}^{2} L_{11}-2 g_{1} g_{2} L_{12}+g_{1}^{2} L_{22}}{2 \omega^{2}}
$$

\section{HELICOIDAL SURFACES IN THE GALILEAN SPACE $G^{3}$}

We will take a regular plane curve $\alpha\left(u_{1}\right)=\left(g\left(u_{1}\right), 0, f\left(u_{1}\right)\right)$ with $g\left(u_{1}\right)>0$ in the $x z$ - plane which is defined on an open interval $I \subset R$. A surface $\Gamma^{2}$ in the Galilean space $G^{3}$ is defined by

$$
\chi\left(u_{1}, u_{2}\right)=\left(g\left(u_{1}\right) \cos \left(u_{2}\right), g\left(u_{1}\right) \sin \left(u_{2}\right), f\left(u_{1}\right)+b u_{2}\right)
$$

is said to be helicoidal surface with axis $o z$, a pitch $b$ and the profile curve $\alpha$.

Without loss of generality, we assume that $\alpha\left(u_{1}\right)=$ $\left(u_{1}, 0, f\left(u_{1}\right)\right)$ is the profile curve in the $x z$ - plane defined on an open interval $I$ of positive real numbers $\left(I \subset R^{+}\right)$. So, the helicoidal surface $\Gamma^{2}$ in $G^{3}$ is given by

$$
\chi\left(u_{1}, u_{2}\right)=\left(u_{1} \cos \left(u_{2}\right), u_{1} \sin \left(u_{2}\right), f\left(u_{1}\right)+b u_{2}\right)
$$

where $f\left(u_{1}\right)$ is a differentiable function defined on $I$.

Theorem 5. Let $\Gamma^{2}$ be helicoidal surface in $G^{3}$ defined by

$$
\chi\left(u_{1}, u_{2}\right)=\left(u_{1} \cos \left(u_{2}\right), u_{1} \sin \left(u_{2}\right), f\left(u_{1}\right)+b u_{2}\right)
$$

where $f\left(u_{1}\right)$ is a differentiable function defined on I. Then the unit normal vector field $N$ of the surface $\Gamma^{2}$ is given by

$$
N=\frac{1}{\omega}\left(0, u_{1} f^{\prime}\left(u_{1}\right) \sin \left(u_{2}\right)+b \cos \left(u_{2}\right),-u_{1}\right)
$$

The first and the second fundamental forms of the surface $\Gamma^{2}$ in $G^{3}$ are given respectively by

$$
d s^{2}=\cos ^{2}\left(u_{2}\right) d u_{1}^{2}-2 u_{1} \sin \left(u_{2}\right) \cos \left(u_{2}\right) d u_{1} d u_{2}+u_{1}^{2} \sin ^{2}\left(u_{2}\right) d u_{2}^{2}(17)
$$

and

$$
I I=\frac{1}{\omega}\left(-u_{1} f^{\prime \prime}\left(u_{1}\right) d u_{1}^{2}+2 b d u_{1} d u_{2}-u_{1}^{2} f^{\prime}\left(u_{1}\right) d u_{2}^{2}\right) .
$$

Proof: Let $\Gamma^{2}$ be helicoidal surface in $G^{3}$ defined by

$$
\chi\left(u_{1}, u_{2}\right)=\left(u_{1} \cos \left(u_{2}\right), u_{1} \sin \left(u_{2}\right), f\left(u_{1}\right)+b u_{2}\right)
$$

Then the unit normal vector field $N$ of the surface $\Gamma^{2}$ is an isotropic vector field defined by

$$
\begin{aligned}
N & =\frac{1}{\omega}\left(0, x_{u_{1}} z_{u_{2}}-x_{u_{2}} z_{u_{1}}, x_{u_{2}} y_{u_{1}}-x_{u_{1}} y_{u_{2}}\right) \\
& =\frac{1}{\omega}\left(0, u_{1} f^{\prime}\left(u_{1}\right) \sin \left(u_{2}\right)+b \cos \left(u_{2}\right),-u_{1}\right)
\end{aligned}
$$

where the positive function $\omega$ is given by

$$
\omega=\sqrt{\left(b \cos \left(u_{2}\right)+u_{1} f^{\prime}\left(u_{1}\right) \sin \left(u_{2}\right)^{2}+u_{1}^{2}\right.}
$$

Here the partial derivatives of the functions $x, y$, and $z$ with respect to $u_{i}(i=1,2)$ are denoted by $x_{u_{i}}, y_{u_{i}}$, and $z_{u_{i}}$, respectively. On the other hand, let us define $g_{i}=x_{u_{i}}, h_{i j}=$ $y_{u_{i}} y_{u_{j}}+z_{u_{i}} z_{u_{j}}, i, j=1,2$. So, the first fundamental form of the surface $\Gamma^{2}$ in $G^{3}$ is given by

$$
d s^{2}=d s_{1}^{2}+\varepsilon d s_{2}^{2}
$$

where

$$
\begin{aligned}
d s_{1}^{2} & =\left(g_{1} d u_{1}+g_{2} d u_{2}\right)^{2} \\
& =\left(\cos \left(u_{2}\right) d u_{1}-u_{1} \sin \left(u_{2}\right) d u_{2}\right)^{2}
\end{aligned}
$$

and

$$
\begin{aligned}
d s_{2}^{2} & =h_{11} d u_{1}^{2}+2 h_{12} d u_{1} d u_{2}+h_{22} d u_{2}^{2} \\
& =\left(\sin ^{2}\left(u_{2}\right)+f^{\prime 2}\left(u_{1}\right)\right) d u_{1}^{2}+2\left(u_{1} \sin \left(u_{2}\right) \cos \left(u_{2}\right)\right. \\
& \left.+b f^{\prime}\left(u_{1}\right)\right) d u_{1} d u_{2}+\left(u_{1} \cos ^{2}\left(u_{2}\right)+b^{2}\right) d u_{2}^{2}
\end{aligned}
$$

Then

$$
d s^{2}=\cos ^{2}\left(u_{2}\right) d u_{1}^{2}-2 u_{1} \sin \left(u_{2}\right) \cos \left(u_{2}\right) d u_{1} d u_{2}+u_{1}^{2} \sin ^{2}\left(u_{2}\right) d u_{2}^{2}
$$

In the sequel, the second fundamental form $I I$ of $\Gamma^{2}$ is given by

$$
I I=\frac{1}{\omega}\left(-u_{1} f^{\prime \prime}\left(u_{1}\right) d u_{1}^{2}+2 b d u_{1} d u_{2}-u_{1}^{2} f^{\prime}\left(u_{1}\right) d u_{2}^{2}\right)
$$

where $L_{11}=\frac{-u_{1}}{\omega} f^{\prime \prime}\left(u_{1}\right), L_{22}=\frac{-u_{1}^{2}}{\omega} f^{\prime}\left(u_{1}\right)$ and $L_{12}=\frac{b}{\omega}$.

Corollary 6. The Gaussian curvature $K$ of the surface $\Gamma^{2}$ is obtained by

$$
K=\frac{1}{\omega^{4}}\left(u_{1}^{3} f^{\prime}\left(u_{1}\right) f^{\prime \prime}\left(u_{1}\right)-b^{2}\right)
$$

Moreover, the mean curvature of the surface $\Gamma^{2}$ is given by

$$
\begin{aligned}
H & =\frac{1}{2 \omega^{3}}\left(-u_{1}^{3} f^{\prime \prime}\left(u_{1}\right) \sin ^{2}\left(u_{2}\right)+2 b u_{1} \sin \left(u_{2}\right) \cos \left(u_{2}\right)\right. \\
& \left.-u_{1}^{2} f^{\prime}\left(u_{1}\right) \cos ^{2}\left(u_{2}\right)\right)
\end{aligned}
$$


Proof: Since the Gaussian curvature $K$ is given by $K=$ $\frac{L_{11} L_{22}-L_{12}^{2}}{\omega^{2}}$, then

$$
\begin{aligned}
K & =\frac{1}{\omega^{2}}\left(\frac{u_{1} f^{\prime \prime}\left(u_{1}\right)}{\omega} \times \frac{u_{1}^{2} f^{\prime}\left(u_{1}\right)}{\omega}-\frac{b^{2}}{\omega^{2}}\right) \\
& =\frac{1}{\omega^{4}}\left(u_{1}^{3} f^{\prime \prime}\left(u_{1}\right) f^{\prime}\left(u_{1}\right)-b^{2}\right)
\end{aligned}
$$

The mean curvature of the surface is obtain from

$$
H=\frac{g_{2}^{2} L_{11}-2 g_{1} g_{2} L_{12}+g_{1}^{2} L_{22}}{2 \omega^{2}}
$$

By substituting, we get

$$
\begin{aligned}
H & =\frac{1}{2 \omega^{2}}\left[\left(-u_{1} \sin \left(u_{2}\right)\right)^{2}\left(\frac{-u_{1} f^{\prime \prime}\left(u_{1}\right)}{\omega}\right)\right. \\
& \left.-2 \cos \left(u_{2}\right)\left(-u_{1} \sin \left(u_{2}\right)\right)\left(\frac{b}{\omega}\right)+\cos ^{2}\left(u_{2}\right)\left(\frac{-u_{1}^{2} f^{\prime}\left(u_{1}\right)}{\omega}\right)\right] \\
& =\frac{1}{2 \omega^{3}}\left[-u_{1}^{3} f^{\prime \prime}\left(u_{1}\right) \sin ^{2}\left(u_{2}\right)+2 b u_{1} \sin \left(u_{2}\right) \cos \left(u_{2}\right)\right. \\
& \left.-u_{1}^{2} f^{\prime}\left(u_{1}\right) \cos ^{2}\left(u_{2}\right)\right]
\end{aligned}
$$

\section{WEIGHTED HELICOIDAL SURFACES IN $G^{3}$}

Let $\Gamma^{2}$ be a helicoidal surface in $G^{3}$ defined by

$$
\chi\left(u_{1}, u_{2}\right)=\left(u_{1} \cos \left(u_{2}\right), u_{1} \sin \left(u_{2}\right), f\left(u_{1}\right)+b u_{2}\right)
$$

where $f\left(u_{1}\right)$ is a differentiable function defined on $I$. Suppose that $\Gamma^{2}$ is the surface in $G^{3}$ with a linear density $e^{\phi}$, where $\phi=$ $\alpha x+\beta y+\gamma z, \alpha, \beta, \gamma$ not all zero.

In this case, the weighted mean curvature $H_{\phi}$ of $\Gamma^{2}$ can be expressed as

$$
H_{\phi}=H-\frac{1}{2}<N, \nabla \phi>_{G^{3}}
$$

where $\nabla \phi$ is the gradient of $\phi$. If $\Gamma^{2}$ is the weighted minimal surface, then

$$
H=\frac{1}{2}<N, \nabla \phi>_{G^{3}}
$$

Theorem 7. Let $\Gamma^{2}$ be weighted minimal helicoidal surface in $G^{3}$ defined by

$$
\chi\left(u_{1}, u_{2}\right)=\left(u_{1} \cos \left(u_{2}\right), u_{1} \sin \left(u_{2}\right), f\left(u_{1}\right)+b u_{2}\right)
$$

with a linear density $e^{\phi}$, then $f\left(u_{1}\right)$ will be one of the following

1. $f\left(u_{1}\right)=\frac{2 b \cot \left(u_{2}\right)}{\cot ^{2}\left(u_{2}\right)-1} \frac{1}{u_{1}}+c_{1} u_{1}^{-\cot ^{2}\left(u_{2}\right)}$

2. $f\left(u_{1}\right)=\frac{1}{2 B}\left[A \ln \left(u_{1}\right)-2 \ln \left(\frac{r\left(u_{1}\right)}{p\left(u_{1}\right)}\right)+\ln (B D)+\ln \left(u_{1}\right)\right]$

3. $f\left(u_{1}\right)$ is the solution of the differential equation

$$
\begin{aligned}
& f^{\prime \prime}\left(u_{1}\right)+\left[\frac{A}{u_{1}}+\frac{B}{u_{1}^{2}}+C\right] f^{\prime}\left(u_{1}\right)+\frac{D}{u_{1}} f^{\prime 2}\left(u_{1}\right)+E f^{\prime 3}\left(u_{1}\right)+\left[\frac{F}{u_{1}}+\right. \\
& \left.\frac{G}{u_{1}^{2}}+\frac{H}{u_{1}^{3}}\right]=0
\end{aligned}
$$

4. $f\left(u_{1}\right)$ is the solution of the differential equation

$$
\begin{aligned}
& f^{\prime \prime}\left(u_{1}\right)+\left(B+\frac{A}{u_{1}}+\frac{C}{u_{1}^{2}}\right) f^{\prime}\left(u_{1}\right)+\left(E+\frac{D}{u_{1}}\right) f^{\prime 2}\left(u_{1}\right)+F f^{\prime 3}\left(u_{1}\right)+ \\
& \left(J+\frac{H}{u_{1}}+\frac{G}{u_{1}^{2}}+\frac{I}{u_{1}^{3}}\right)=0
\end{aligned}
$$

Proof: Let $\Gamma^{2}$ be a helicoidal surface in $G^{3}$ defined by

$$
\chi\left(u_{1}, u_{2}\right)=\left(u_{1} \cos \left(u_{2}\right), u_{1} \sin \left(u_{2}\right), f\left(u_{1}\right)+b u_{2}\right)
$$

where $f\left(u_{1}\right)$ is a differentiable function defined on I. By substituting in equation (27) we obtain

$$
\begin{aligned}
& -u_{1}^{3} f^{\prime \prime}\left(u_{1}\right) \sin ^{2}\left(u_{2}\right)+2 b u_{1} \sin \left(u_{2}\right) \cos \left(u_{2}\right)-u_{1}^{2} f^{\prime}\left(u_{1}\right) \cos ^{2}\left(u_{2}\right) \\
& =\left(\left(b \cos \left(u_{2}\right)+u_{1} f^{\prime}\left(u_{1}\right) \sin \left(u_{2}\right)\right)^{2}+u_{1}^{2}\right)<\left(0, u_{1} f^{\prime}\left(u_{1}\right) \sin \left(u_{2}\right)\right. \\
& \left.+b \cos \left(u_{2}\right),-u_{1}\right),(\alpha, \beta, \gamma)>
\end{aligned}
$$

Now, we can distinguish two cases according to the value of $\alpha$. Case 1. If $\alpha \neq 0$

In this case the vector $(\alpha, \beta, \gamma)$ is non-isotropic, with some simple calculation we can obtain the following differential equation

$$
f^{\prime \prime}\left(u_{1}\right)+\frac{1}{u_{1}} \cot ^{2}\left(u_{2}\right) f^{\prime}\left(u_{1}\right)=\frac{2 b}{u_{1}^{2}} \cot \left(u_{2}\right)
$$

To solve this equation, we make reduction of the order as: Let $f^{\prime}\left(u_{1}\right)=y\left(u_{1}\right)$ which gives $f^{\prime \prime}\left(u_{1}\right)=y^{\prime}\left(u_{1}\right)$, substitutes into equation (31) we obtain the differential equation

$$
y^{\prime}\left(u_{1}\right)+\frac{1}{u_{1}} \cot ^{2}\left(u_{2}\right) y\left(u_{1}\right)=\frac{2 b}{u_{1}^{2}} \cot \left(u_{2}\right)
$$

Integrating factor $I F=u_{1}^{\cot ^{2}\left(u_{2}\right)}$ and hence the solution is given by

$$
y\left(u_{1}\right)=\frac{2 b \cot \left(u_{2}\right)}{\cot ^{2}\left(u_{2}\right)-1} \frac{1}{u_{1}}+c_{1} u_{1}^{-\cot ^{2}\left(u_{2}\right)}
$$

i.e.,

$$
f^{\prime}\left(u_{1}\right)=\frac{2 b \cot \left(u_{2}\right)}{\cot ^{2}\left(u_{2}\right)-1} \frac{1}{u_{1}}+c_{1} u_{1}^{-\cot ^{2}\left(u_{2}\right)}
$$

which gives

$$
f\left(u_{1}\right)=\frac{2 b \cot \left(u_{2}\right)}{\cot ^{2}\left(u_{2}\right)-1} \ln \left(u_{1}\right)+\frac{c_{1}}{1-\cot ^{2}\left(u_{2}\right)} u_{1}^{1-\cot ^{2}\left(u_{2}\right)}+c_{2}
$$

Therefore, $\Gamma^{2}$ is determined by

$$
\begin{array}{r}
\chi\left(u_{1}, u_{2}\right)=\left(u_{1} \cos \left(u_{2}\right), u_{1} \sin \left(u_{2}\right), \frac{2 b \cot \left(u_{2}\right)}{\cot ^{2}\left(u_{2}\right)-1} \ln \left(u_{1}\right)\right. \\
\left.+\frac{c_{1}}{1-\cot ^{2}\left(u_{2}\right)} u_{1}^{1-\cot ^{2}\left(u_{2}\right)}+b u_{2}+c_{2}\right)
\end{array}
$$

where

$$
\begin{aligned}
& \quad z\left(u_{1}, u_{2}\right)=\frac{2 b \cot \left(u_{2}\right)}{\cot ^{2}\left(u_{2}\right)-1} \ln \left(u_{1}\right)+\frac{c_{1}}{1-\cot ^{2}\left(u_{2}\right)} u_{1}^{1-\cot ^{2}\left(u_{2}\right)} \\
& +b u_{2}+c_{2}
\end{aligned}
$$


$c_{1}, c_{2}$ are constants.

Case 2. If $\alpha=0$

In this case the vector $(0, \beta, \gamma)$ is an isotropic and as before, we obtain

$$
\begin{aligned}
& \frac{1}{\omega^{2}}\left(-u_{1}^{3} f^{\prime \prime}\left(u_{1}\right) \sin ^{2}\left(u_{2}\right)+2 b u_{1} \sin \left(u_{2}\right) \cos \left(u_{2}\right)\right. \\
- & \left.u_{1}^{2} f^{\prime}\left(u_{1}\right) \cos ^{2}\left(u_{2}\right)\right) \\
= & \beta u_{1} f^{\prime}\left(u_{1}\right) \sin \left(u_{2}\right)+\beta b \cos \left(u_{2}\right)-\gamma u_{1}
\end{aligned}
$$

Case 2.1. If $\beta=0$, therefore

$$
\begin{array}{r}
\frac{1}{\omega^{2}}\left(-u_{1}^{3} f^{\prime \prime}\left(u_{1}\right) \sin ^{2}\left(u_{2}\right)+2 b u_{1} \sin \left(u_{2}\right) \cos \left(u_{2}\right)\right. \\
\left.-u_{1}^{2} f^{\prime}\left(u_{1}\right) \cos ^{2}\left(u_{2}\right)\right)=-\gamma u_{1}
\end{array}
$$

which gives the following differential equation

$$
f^{\prime \prime}\left(u_{1}\right)+A \frac{f^{\prime}\left(u_{1}\right)}{u_{1}}-B f^{\prime 2}\left(u_{1}\right)=\frac{C}{u_{1}^{2}}+D
$$

where $A=\cot ^{2}\left(u_{2}\right)-2 b \gamma \cot \left(u_{2}\right), B=\gamma, C=\gamma b^{2} \cot ^{2}\left(u_{2}\right)+$ $2 b \cot \left(u_{2}\right), D=\frac{\gamma}{\sin ^{2}\left(u_{2}\right)}$.

By using Bessel's functions of first and second order, a simple computations gives that the solution of Equation (39) can be written in the form

$$
f\left(u_{1}\right)=\frac{1}{2 B}\left[A \ln \left(u_{1}\right)-2 \ln \left(\frac{r\left(u_{1}\right)}{p\left(u_{1}\right)}\right)+\ln (B D)+\ln \left(u_{1}\right)\right]
$$

such that

$$
\begin{array}{r}
r\left(u_{1}\right)=B\left[c_{2} Y_{n}\left(\sqrt{B D} u_{1}\right)-c_{1} J_{n}\left(\sqrt{B D} u_{1}\right)\right] \\
p\left(u_{1}\right)=\left[J_{n+1}\left(\sqrt{B D} u_{1}\right) Y_{n}\left(\sqrt{B D} u_{1}\right)\right. \\
\left.-J_{n}\left(\sqrt{B D} u_{1}\right) Y_{n+1}\left(\sqrt{B D} u_{1}\right)\right]
\end{array}
$$

and

$$
n=\frac{1}{2} \sqrt{A^{2}-4 B C-2 A+1}
$$

with an arbitrary constants $c_{1}, c_{2}$. Therefore, in this case, the surface $\Gamma^{2}$ is given by

$$
\begin{array}{r}
\chi\left(u_{1}, u_{2}\right)=\left(u_{1} \cos \left(u_{2}\right), u_{1} \sin \left(u_{2}\right), \frac{1}{2 B}\left[A \ln \left(u_{1}\right)\right.\right. \\
\left.\left.-2 \ln \left(\frac{r\left(u_{1}\right)}{p\left(u_{1}\right)}\right)+\ln (B D)+\ln \left(u_{1}\right)\right]+b u_{2}\right)
\end{array}
$$

Case 2.2. If $\gamma=0$, then

$$
\begin{aligned}
& -u_{1}^{3} f^{\prime \prime}\left(u_{1}\right) \sin ^{2}\left(u_{2}\right)+2 b u_{1} \sin \left(u_{2}\right) \cos \left(u_{2}\right)-u_{1}^{2} f^{\prime}\left(u_{1}\right) \cos ^{2}\left(u_{2}\right) \\
& =\omega^{2}\left(\beta u_{1} f^{\prime}\left(u_{1}\right) \sin \left(u_{2}\right)+\beta b \cos \left(u_{2}\right)\right)
\end{aligned}
$$

a simple computations gives the next differential equation

$$
\begin{aligned}
& f^{\prime \prime}\left(u_{1}\right)+\left[\frac{A}{u_{1}}+\frac{B}{u_{1}^{2}}+C\right] f^{\prime}\left(u_{1}\right)+\frac{D}{u_{1}} f^{\prime 2}\left(u_{1}\right) \\
& +E f^{\prime 3}\left(u_{1}\right)+\left[\frac{F}{u_{1}}+\frac{G}{u_{1}^{2}}+\frac{H}{u_{1}^{3}}\right]=0
\end{aligned}
$$

where $A=\cot ^{2}\left(u_{2}\right), B=3 \beta b^{2} \cos \left(u_{2}\right) \cot \left(u_{2}\right), C=\frac{\beta}{\sin \left(u_{2}\right)}$, $D=3 \beta b \cos \left(u_{2}\right), E=\beta \sin \left(u_{2}\right), F=\frac{\beta b \cot \left(u_{2}\right)}{\sin \left(u_{2}\right)}, G=-2 b \cot \left(u_{2}\right)$ and $H=\beta b^{3} \cos \left(u_{2}\right) \cot ^{2}\left(u_{2}\right)$.

Case 2.3. If $\gamma \beta \neq 0$, therefore

$$
\begin{aligned}
& -u_{1}^{3} f^{\prime \prime}\left(u_{1}\right) \sin ^{2}\left(u_{2}\right)+2 b u_{1} \sin \left(u_{2}\right) \cos \left(u_{2}\right)-u_{1}^{2} f^{\prime}\left(u_{1}\right) \cos ^{2}\left(u_{2}\right) \\
& =\omega^{2}\left(\beta u_{1} f^{\prime}\left(u_{1}\right) \sin \left(u_{2}\right)+\beta b \cos \left(u_{2}\right)-\gamma u_{1}\right)
\end{aligned}
$$

which gives the following differential equation

$$
\begin{aligned}
& f^{\prime \prime}\left(u_{1}\right)+\left(B+\frac{A}{u_{1}}+\frac{C}{u_{1}^{2}}\right) f^{\prime}\left(u_{1}\right)+\left(E+\frac{D}{u_{1}}\right) f^{\prime 2}\left(u_{1}\right)+F f^{\prime 3}\left(u_{1}\right) \\
& +\left(J+\frac{H}{u_{1}}+\frac{G}{u_{1}^{2}}+\frac{I}{u_{1}^{3}}\right)=0
\end{aligned}
$$

where $A=\cot ^{2}\left(u_{2}\right)-2 \gamma b \cot \left(u_{2}\right), B=\frac{\beta}{\sin \left(u_{2}\right)}, C=$ $\frac{3 \beta b^{2} \cos ^{2}\left(u_{2}\right)}{\sin \left(u_{2}\right)}, D=3 \beta b \cos \left(u_{2}\right), E=-\gamma, F=\beta \sin \left(u_{2}\right), G=$ $-\left(2 b \cot \left(u_{2}\right)+\gamma b^{2} \cot ^{2}\left(u_{2}\right)\right), H=\frac{\beta b \cos \left(u_{2}\right)}{\sin ^{2}\left(u_{2}\right)}, I=\frac{\beta b^{3} \cos ^{3}\left(u_{2}\right)}{\sin ^{2}\left(u_{2}\right)}$, and $J=-\frac{\gamma}{\sin ^{2}\left(u_{2}\right)}$.

\section{CONCLUSION AND FURTHER RESEARCH}

In this work, we constructed helicoidal surfaces in the Galilean 3-space and studied the First and the Second Fundamental Forms. Moreover, we calculated mean and Gaussian curvature for such surfaces. Also, we considered the Galilean 3-space with a linear density $e^{\phi}, \phi=\alpha x+\beta y+\gamma z$ such that $\alpha, \beta, \gamma$ not all zero and constructed a weighted helicoidal surface by solving a second order non-linear differential equation. Moreover, we discussed an explicit parametrization for the helicoidal surfaces in $G^{3}$.

Analogously to how a Minkowski 3-space relates to a Euclidean 3-space, one has the notion of Pseudo-Galilean 3 -space $G_{1}^{3}$. As known, $G_{1}^{3}$ is similar to $G^{3}$, but the Pseudo-Galilean scalar product of two vectors $r=\left(r_{1}, r_{2}, r_{3}\right)$ and $s=\left(s_{1}, s_{2}, s_{3}\right)$ is defined by

$$
<r, s>= \begin{cases}r_{1} s_{1}, & \text { if } r_{1} \neq 0 \text { or } s_{1} \neq 0 \\ r_{2} s_{2}-r_{3} s_{3}, & \text { if } r_{1}=s_{1}=0\end{cases}
$$

Therefore, there exist four types of isotropic vectors $r=\left(0, r_{2}, r_{3}\right)$ in $G_{1}^{3}$ : spacelike vectors (if $r_{2}^{2}-r_{3}^{2}>0$ ), timelike vectors (if $r_{2}^{2}-r_{3}^{2}<0$ ) and two types of lightlike vectors (if $r_{2}= \pm r_{3}$ ) [15]. Thus, one can define different types of Helicoidal surfaces in $G_{1}^{3}$.

\section{DATA AVAILABILITY STATEMENT}

All datasets generated for this study are included in the article/supplementary material. 


\section{AUTHOR CONTRIBUTIONS}

All authors listed have made a substantial, direct and intellectual contribution to the work, and approved it for publication.

\section{REFERENCES}

1. Morgan F. Manifolds with density. Notices Am Math Soc. (2005) 52:853-8.

2. Hieu DT, Hoang NM. Ruled minimal surfaces in $R^{3}$ with density $e^{z}$. Pac J Math. (2009) 243:277-85. doi: 10.2140/pjm.2020.305-1

3. Kenmotsu K. Surface of revolution with prescribed mean curvature. Tohoku Math J. (1980) 32:147-53. doi: 10.2748/tmj/1178229688

4. Baikoussis C, Koufogiorgos T. Helicoidal surface with prescribed mean or Gaussian curvature. J Geom. (1998) 63:25-9.

5. Beneki CC, Kaimakamis G, Papantoniou BJ. Helicoidal surfaces in threedimensional Minkowski space. J Math Anal Appl. (2002) 275:586-614. doi: 10.1016/S0022-247X(02)00269-X

6. Ji F, Hou ZH. A kind of helicoidal surfaces in 3-dimensional Minkowski space. J Math Anal Appl. (2005) 304:632-43. doi: 10.1016/j.jmaa.2004.09.065

7. Kim DS, Kim YH, Lee JW. Construction of helicoidal surfaces in Euclidean space with density. Symmetry. (2017) 9:173-81. doi: 10.3390/sym9090173

8. Agarwal P, Dragomir SS, Jleli M, Samet B. Advances in Mathematical Inequalities and Applications. Birkhauser; Springer Nature Singapore Pte Ltd. (2018).

9. Hieu DT, Nam TL.The classification of constant weighted curvature curves in the plane with a log-linear density. Am Inst Math Sci. (2014) 13:1641-52. doi: 10.3934/cpaa.2014.13.1641

10. Gromov M. Isoperimetry of waists and concentration of maps. Geom Funct Anal. (2003) 13:178-215. doi: 10.1007/s000390300004

11. Shang Y. Lack of Gromov-hyperbolicity in small-world networks. Central Eur J Math. (2012) 10:1152-8. doi: 10.2478/s11533-012-0032-8

12. Yoon DW. Weighted minimal translation surfaces in the Galilean space with density. Open Math. (2017) 15:459-66. doi: 10.1515/math-2017-0043

\section{ACKNOWLEDGMENTS}

The authors wish to express their sincere thanks to referee for making several useful comments that improved the first version of the paper.

13. Belarbi L, Belkhelfa M. Surfaces in $\mathrm{R}^{3}$ with density. i-managers J Math. (2012) 1:34-48. doi: 10.26634/jmat1.1.1845

14. Dede M, Ekici C. On parallel ruled surfaces in Galilean space. Kragujevac J Math. (2016) 40:47-59. doi: 10.5937/KgJMath1601047D

15. Dede M, Ekici C, Goemans W. Surfaces of Revolution with vanishing curvature in Galilean 3-space. J Math Phys Anal Geom. (2018) 14:141-52. doi: $10.15407 / \operatorname{mag} 14.02 .141$

16. ELzawy M, Mosa S. Razzaboni surfaces in the Galilean space $G^{3}$. Far East J Math Sci. (2018) 108:13-26. doi: 10.17654/MS1080 10013

17. Yoon DW, Lee JW, Lee CW. $\phi$-minimal rotational surfaces in Pseudo-Galilean space with density. Ann Polonici Math. (2017) 120. doi: 10.4064/ap170406-21-10

18. Aydin ME, Ogrenmis AO. Spherical product surfaces in the Galilean space Konuralp J Math. (2016) 4:290-8.

19. Ozturk U, Ozturk EBK, Nesovic E. On equiform Darboux helices in Galilean 3-space. Math Commun. (2018) 23:145-59.

Conflict of Interest: The authors declare that the research was conducted in the absence of any commercial or financial relationships that could be construed as a potential conflict of interest.

Copyright (c) 2020 Mosa and Elzawy. This is an open-access article distributed under the terms of the Creative Commons Attribution License (CC BY). The use, distribution or reproduction in other forums is permitted, provided the original author(s) and the copyright owner(s) are credited and that the original publication in this journal is cited, in accordance with accepted academic practice. No use, distribution or reproduction is permitted which does not comply with these terms. 